\title{
Spontaneous Coronary Artery Dissection
}

\author{
- From Diagnosis to Management -
}

\author{
Fernando Alfonso, MD, PhD; Teresa Bastante, MD; Fernando Rivero, MD; Javier Cuesta, MD; \\ Amparo Benedicto, MD; Jacqueline Saw, MD; Rajiv Gulati, MD, PhD
}

\begin{abstract}
Spontaneous coronary artery dissection (SCAD) is a rare but challenging clinical entity of unknown etiology. From a pathophysiological standpoint, SCAD may occur in patients with a coronary intimal tear (presenting with the classic angiographic "flap" and multiple lumens), but also in patients without an intimal rupture (presenting as an intramural hematoma). Until now, available information on SCAD was largely based on multiple, small case-series studies but, recently, data from relatively large registries have cast a new light on this disease. Classically, SCAD was thought to present in young females without traditional atherosclerotic risk factors but recent reports suggest a broader clinical spectrum encompassing older patients with associated coronary artery disease. In this review, we concentrate on 3 main aspects of this unique disease: (1) the value of intracoronary diagnostic techniques (intravascular ultrasound and optical coherence tomography) to complement coronary angiography and to provide novel diagnostic insights on this elusive clinical condition; (2) the growing clinical evidence suggesting an association and potential causation between fibromuscular dysplasia and SCAD; and (3) the challenges of coronary revascularization in this adverse anatomic setting, together with recent data suggesting that a initial, conservative medical management may be preferable for the majority of patients with SCAD. (Circ J 2014; 78: 2099-2110)
\end{abstract}

Key Words: Acute coronary syndrome; Coronary revascularization; Fibromuscular dysplasia; Intracoronary diagnostic imaging; Spontaneous coronary artery dissection

$\mathbf{I}$ n early reports, the diagnosis of spontaneous coronary artery dissection (SCAD) was only established postmortem. ${ }^{1,2}$ These scarce preliminary reports based on autopsy findings after episodes of sudden cardiac death suggested the condition had a dismal prognosis., ${ }^{1,2}$ Interestingly, an intimal tear was seldom found in the early autopsy series. Back in 1931, Pretty published the first patient with SCAD, a 42-year-old woman who died suddenly after severe retching and vomiting induced a ruptured right coronary artery (RCA) atheroma. ${ }^{1}$ The first angiographic diagnosis was reported by Forker et al in 1969 in a 56-year-old male patient with SCAD of the RCA that eventually required coronary artery bypass grafting (CABG). ${ }^{3}$ Subsequently, many small, retrospective case-series with a limited clinical follow-up were published. ${ }^{4-18}$ However, only very recently, relatively large series of patients providing additional information on this unique disease have been published. ${ }^{19-22}$ Altogether, $<700$ patients with SCAD have been reported in the literature to date. ${ }^{15-18}$

\section{Clinical Characterization of SCAD}

\section{Incidence and Prevalence}

SCAD remains a rare and challenging clinical condition, with an estimated prevalence ranging from $0.1 \%$ to $1.1 \%$ in most angiographic series. ${ }^{15-18}$ However, there are major reasons to believe that the prevalence and incidence of SCAD have been largely underestimated, including: (1) initial presentation as sudden death, (2) underuse of coronary angiography (CAG) in young females with chest pain, and (3) the well-known inherent limitations of CAG to detect disease of the vessel wall. Early studies suggested a very high prevalence of female patients, although recent series that included patients with associated atherosclerotic coronary artery disease (CAD) have found a male predominance. ${ }^{4-13}$ Studies that excluded atheromatous forms of SCAD have found a predominance of women. The most frequent age of presentation is at the 5 th or 6th decade of life. ${ }^{4-13}$ However, it remains important to highlight that SCAD may occur in very young patients. Accordingly, this diagnosis should be always contemplated in young patients, particularly women, presenting with acute coronary syndrome (ACS). ${ }^{15}$ Most patients present with ACS, including ST-segment eleva-

Received July 17, 2014; accepted July 18, 2014; released online August 13, 2014

Cardiac Department, Hospital Universitario de La Princesa, Madrid (F.A., T.B., F.R., J.C., A.B.), Spain; Division of Cardiology, Vancouver General Hospital, University of British Columbia, Vancouver (J.S.), Canada; and Division of Cardiovascular Diseases, Mayo Clinic, Rochester, MN (R.G.), USA

Mailing address: Fernando Alfonso, MD, PhD, FESC, Cardiac Department, Hospital Universitario de La Princesa, IIS-IP, Universidad Autónoma de Madrid, C/Diego de León 62, Madrid 28006, Spain. E-mail: falf@ hotmail.com

ISSN-1346-9843 doi:10.1253/circj.CJ-14-0773

All rights are reserved to the Japanese Circulation Society. For permissions, please e-mail: cj@j-circ.or.jp 


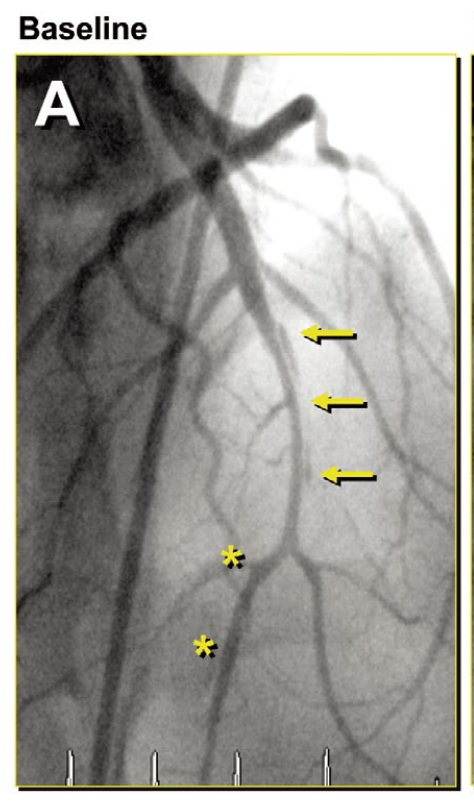

Post Stent

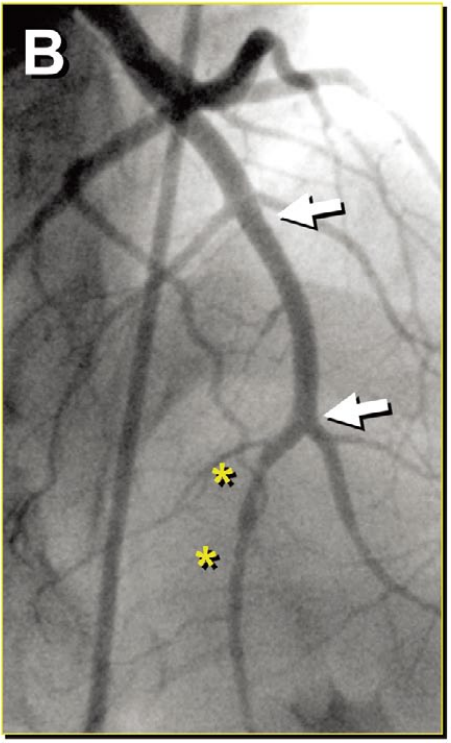

Late Follow-up

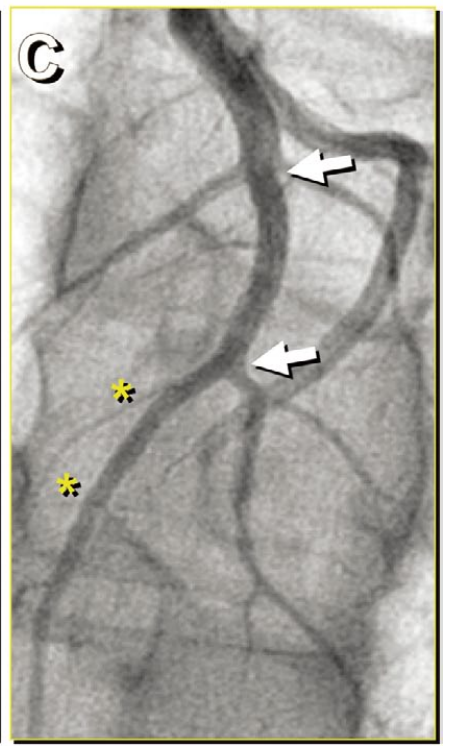

Figure 1. Spontaneous coronary artery dissection affecting the left anterior descending coronary artery. (A) Characteristic angiographic flap is readily visualized (small arrows). (B) After stenting (arrows) the angiographic image improves, although a diffuse narrowing of the distal vessel $\left(^{*}\right)$ is visualized. (C) At late follow-up, the stented site shows an excellent angiographic result and a significant improvement of the vessel caliber is detected in the distal vessel $\left({ }^{*}\right)$. Reproduced from Alfonso $\mathrm{F}$ et al ${ }^{19}$ with permission from the American College of Cardiology Foundation.

tion myocardial infarction (STEMI). ${ }^{4-13}$ Once the initial clinical episode has been stabilized, prognosis tends to be favorable. Classically, SCAD has been associated with several specific clinical situations, including pregnancy, the peripartum and perimenopausal periods, use of oral contraceptives, heavy isometric exercise, and also with systemic connective tissue disorders (Ehlers-Danlos syndrome, Marfan syndrome and systemic lupus erythematosus). ${ }^{14-18}$

\section{Pathogenesis}

The pathogenesis of SCAD has not been settled and appears to be multifaceted. Two main pathological subsets have been described: with and without an intimal rupture. ${ }^{14-18}$ An intimal tear may precipitate bleeding into the wall, with free communication between the true and false coronary lumens. Alternatively, a primary disruption of the "vasa vasorum" with subsequent bleeding and intramedial hemorrhage has been proposed as the underlying mechanism in patients in whom an intimal tear could not be identified. The resulting intramural hematoma has no communication with the coronary lumen. ${ }^{14-18}$ Interestingly, pathologic studies have demonstrated that the dissection plane often lies within the outer third of the media. Pressure-driven expansion of the false lumen leads to axial propagation of the disease and true lumen compression, resulting in myocardial ischemia. ${ }^{14-18}$ Alternatively, in patients with atherosclerotic CAD, the rupture of a thin-cap fibroatheroma might lead to SCAD. However, this appears to be a rare cause of SCAD, as only in a limited anatomic setting would these plaque ruptures progress longitudinally along the vessel for a significant extent. In fact, lessons learnt from the field of interventional cardiology suggest that the presence of atherosclerotic plaque within the vessel wall halts the progression of coronary dissections. It has been hypothesized that scarring from atherosclerosis limits the extension of dissection. Accordingly, most investigators currently accept that in patients with atherosclerotic CAD the underlying pathology is substantially different from that seen in patients with non-atherosclerotic SCAD; ${ }^{14-18}$ in fact, the term "SCAD" generally implies and is synonymous with "non-atherosclerotic SCAD".

Major attention has been focused on further characterization of the underlying anatomical substrate causing a "vulnerable" coronary vessel wall. ${ }^{14-18}$ Classical studies suggest that pregnancy and postpartum are high-risk periods in young females. Structural changes in the arterial wall related to hormonal changes, coupled with the hemodynamic stress secondary to the increase in blood volume, together with the prothrombotic milieu characteristic of pregnancy, were considered to predispose to SCAD. ${ }^{14-18}$ Estrogen modifies the arterial wall architecture, inducing hypertrophy of the smooth muscle cells, loosens the intercellular matrix with an increase in acid mucopolysaccharides and decrease in collagen production. ${ }^{14-18}$ Alternatively, in patients with connective tissue disorders, medial degeneration of the coronary wall is thought to explain the arterial weakness. Accumulation of eosinophilic infiltrates has been described in patients with SCAD. Interestingly, the cytotoxic activity of these cells might lead to cystic medial necrosis. ${ }^{23}$ Conversely, eosinophilic infiltrates might only be innocent bystanders or a non-specific secondary inflammatory reaction. Recent reports suggesting an association between SCAD and fibromuscular dysplasia (FMD) in large arteries will be addressed in detail later. ${ }^{22}$ SCAD diagnosis requires absence of iatrogenic catheter-induced or other traumatic etiology. ${ }^{24,25} \mathrm{How}-$ ever, the importance of triggers has also been emphasized by many investigators, suggesting that local shear stress may play 


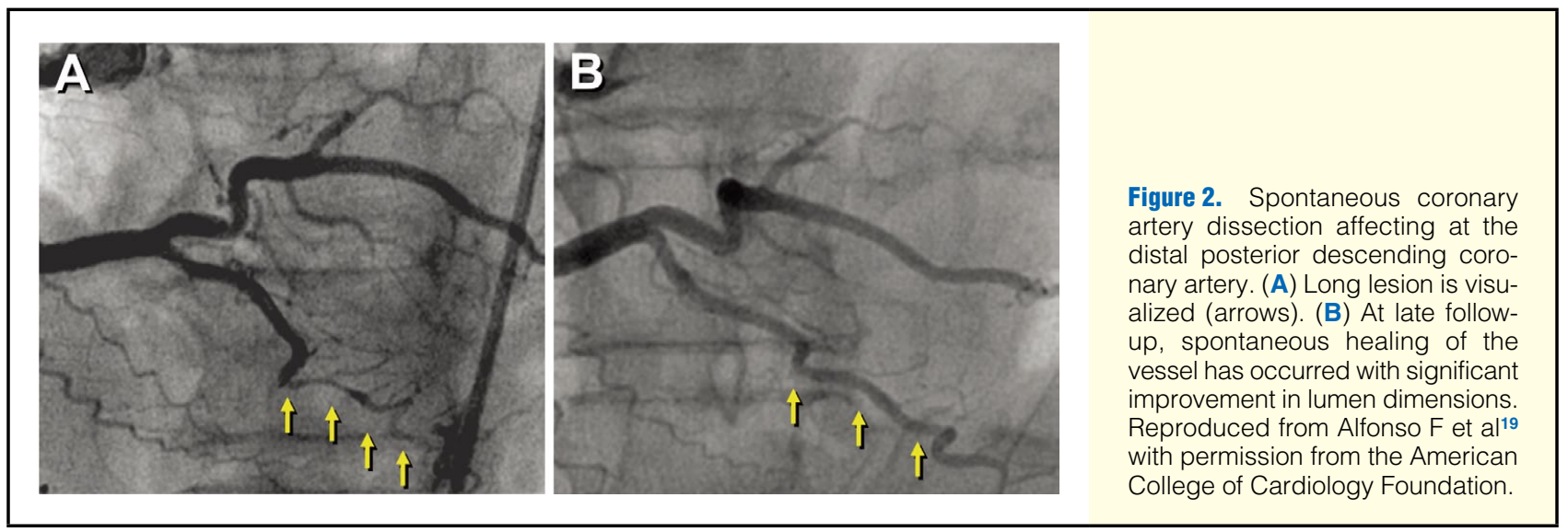

a major role. In this regard, vigorous physical exercise, repeat coughing or squeezing, blunt chest trauma, cocaine abuse and coronary spasm have all been reported as potential triggers of SCAD. ${ }^{14-18}$

\section{Diagnosis}

CAG remains the main diagnostic tool for the diagnosis of SCAD. ${ }^{4-13}$ Angiographically, SCAD is diagnosed in patients presenting with a longitudinal filling defect (the "intimal flap") that generates a double lumen. In fact, the radiolucent flap is considered the angiographic hallmark of $\mathrm{SCAD}^{4-13}$ (Figure 1). Alternatively, delayed contrast clearance suggests slow flow in the false lumen. The left anterior descending coronary artery (LAD) is the most frequently involved vessel. Although the NHLBI classification was devised for "iatrogenic" dissections generated during coronary interventions, this classification scheme is also used for patients with SCAD. However, the diagnosis of SCAD can easily be overlooked on angiography. ${ }^{15}$ Therefore, a meticulous analysis of the coronary angiogram by experienced operators remains of upmost importance. Full-lumen opacification from adequately sized and well (but carefully) engaged catheters remains critical. Intracoronary nitroglycerin should be used to enlarge the reference vessel segments and to disclose subtle luminal abnormalities. Multiple views should be performed to better delineate indirect signs of lumen compromise and to disclose the angiographic flap. Notably, the widespread use of early angiography in patients with ACS has promoted an increase in the diagnosis of SCAD. A diffuse lesion in a young patient with smooth appearance in the remaining coronary arteries should always raise the clinical suspicious of an underlying intramural hematoma (Figures 2,3). ${ }^{15}$ A simple angiographic classification of SCAD and an algorithm to improve the diagnosis of SCAD were described by Saw. ${ }^{16}$ Lesions with the hallmark multiple lumen and/or contrast wall stain were classified as type 1 SCAD. Lesions with long, diffuse smooth stenosis (representing intramural hematoma) were classified as type 2 SCAD. And lesions with focal or tubular stenosis (because of intramural hematoma) that mimics atherosclerosis were classified as type 3 SCAD. Intracoronary imaging is required to make the diagnosis of SCAD in the type 3 angiographic form, and is very helpful in type 2 angiographic form. In the next section, we will review in detail the value of intracoronary imaging to aid SCAD diagnosis.

Surprisingly, however, the diagnostic value of multislice computed tomography (MSCT) in patients with SCAD has not

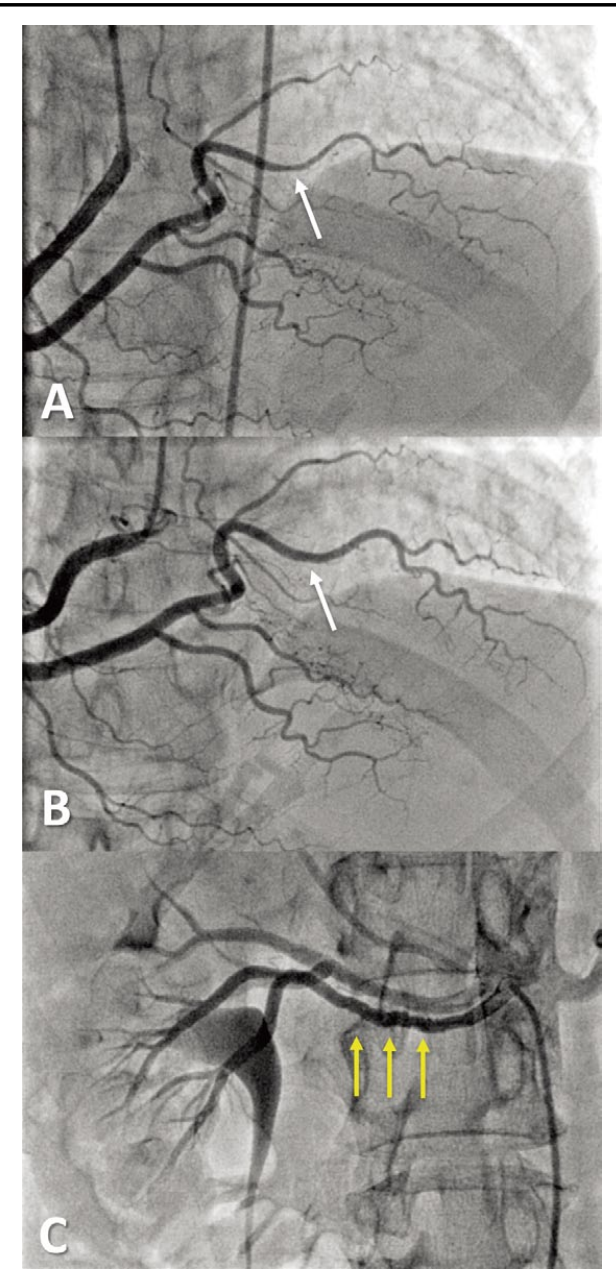

Figure 3. Patient presenting with an intramural hematoma of the distal right coronary artery and concomitant fibromuscular dysplasia (FMD) of the renal artery. (A) Type 2 angiographic spontaneous coronary artery dissection (SCAD) with diffuse smooth narrowing of the right posterolateral branch starting from the arrow. (B) Repeat angiogram 13 months later showing complete angiographic resolution of the image at the right posterolateral branch. (C) Selective angiogram of the right renal artery showing irregular beading of the mid-segment (arrow) of the main renal artery, representing FMD in this woman with SCAD. 


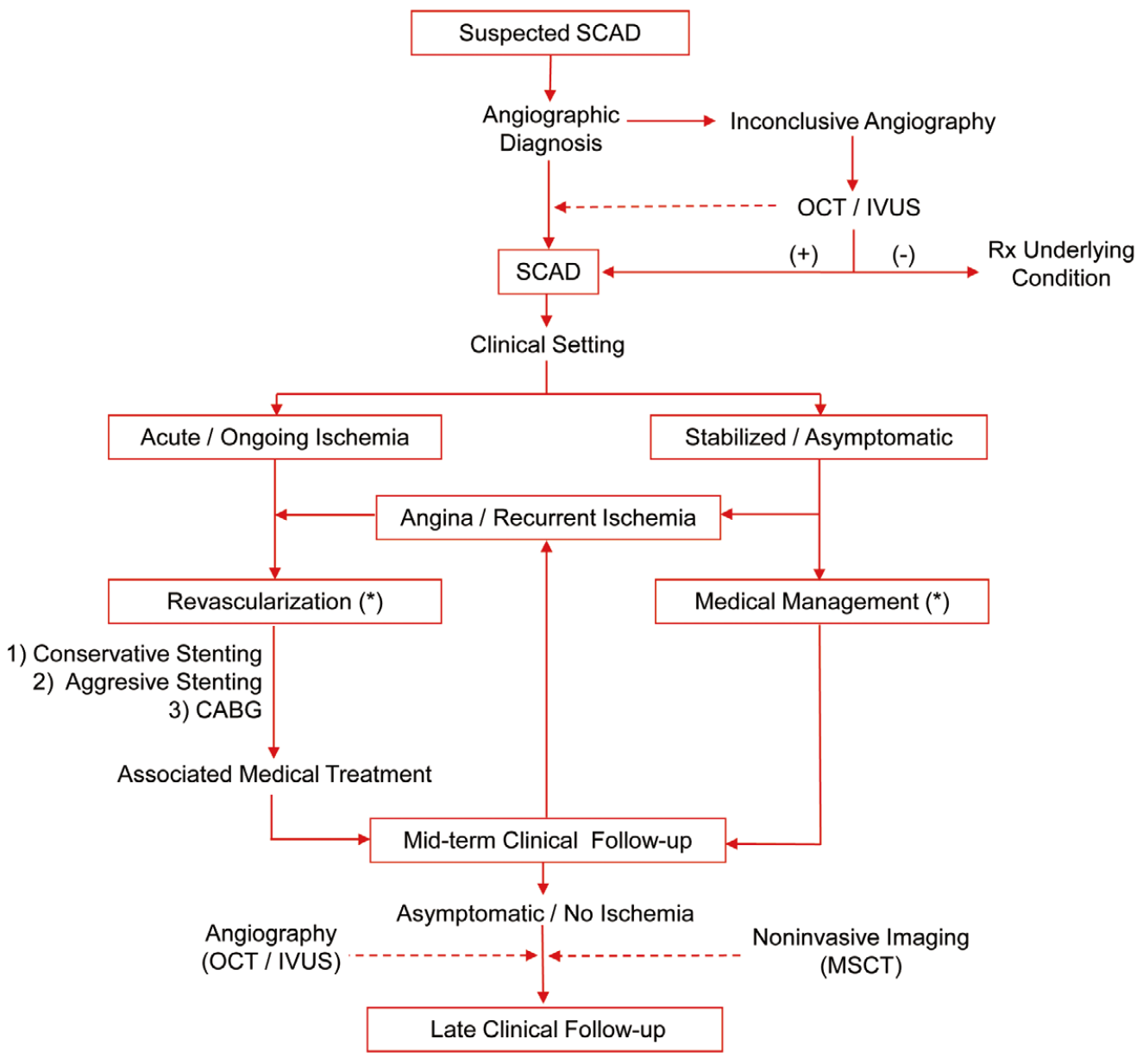

Figure 4. Diagnostic and management algorithm. Broken lines denote suggestions for selected patients or with research purposes. CABG, coronary artery bypass grafting; IVUS, Intravascular ultrasound; MSCT, multislice computed tomography; OCT, Optical coherence tomography; SCAD, spontaneous coronary artery dissection. (*) Revascularization is not indicated in small or distal vessels. Reproduced from Alfonso F et al19 with permission from the American College of Cardiology Foundation.

been fully elucidated. ${ }^{26,27}$ In selected cases, however, a double lumen or an intramural hematoma (presenting as a low-density signal surrounding the lumen) can be demonstrated on MSCT. ${ }^{26,27}$ Patients with SCAD in large proximal vessels appear ideal candidates for this technology. However, in the majority of cases, SCAD affects the mid to distal segments of the coronary arteries and side-branches, rendering MSCT inadequate for the diagnosis of most SCAD cases, because the spatial resolution remains limited and the assessment of distal or small vessels is challenging. In selected cases of large vessel involvement, MSCT may be suited for follow-up purposes, providing a dynamic picture of the natural history of this disease. ${ }^{26,27}$

\section{Treatment}

Eight decades after the initial pathological description, ${ }^{1}$ management of patients with SCAD remains empirical and controversial, ${ }^{14-18}$ because of the lack of comparative studies of different therapeutic strategies. ${ }^{4-13,19-22}$ In particular, there is a lack of scientific evidence supporting the value of specific pharmacological regimens or the value of systematic coronary revascularization over conservative medical management. Classically, thrombolytics and glycoprotein IIb/IIIa inhibitors are considered to be contraindicated. ${ }^{15}$ Thrombolytics may promote additional intramural bleeding; nevertheless, many SCAD patients presenting with STEMI actually receive thrombolytics before diagnostic angiography. ${ }^{14-18}$ Other investigators have reported no deleterious effect of the use of glycoprotein IIb/ IIIa inhibitors in these patients. ${ }^{15}$ However, potent antiplatelet drugs and anticoagulants may act as a double-edged sword in this challenging condition. ${ }^{14-18}$ These agents may prevent progressive thrombus formation, eventually leading to vessel occlusion. Alternatively, by deferring false lumen thrombosis and preventing vessel healing, they may facilitate progression and extension of the disease. Nevertheless, aggressive antiplatelet therapy seems reasonable in patients with a large thrombus burden in the true lumen. The use of aspirin and clopidogrel following SCAD presentation is advocated by some, but remains unstudied in clinical trials. ${ }^{17}$ Likewise, calcium-channel blockers are recommended in patients with vasospastic vessels. Beta-blockers are considered the mainstay of therapy. ${ }^{14-18}$ Beta-blockers reduce oxygen consumption and local shear 
stress, with the potential to stabilize the condition acutely and prevent late recurrence. Overall, the medical treatment for SCAD is similar to that recommended for patients with ACS. However, the value of the novel more potent antiplatelet agents (prasugrel and ticagrelor) in these patients remains unknown. HMG CoA reductase inhibitors ("statins"), while being a mainstay agent in typical atherosclerotic ACS, are untested in SCAD. In fact, 1 study suggested a higher risk of recurrent SCAD in patients who were discharged on statin therapy after a first SCAD episode. ${ }^{21}$ This observation, derived from a retrospective analysis, may have been confounded, but underscores the lack of prospective controlled data to guide pharmacotherapy in SCAD-related ACS.

Revascularization is shadowed by the fragility of the vessel wall, which limits both surgical and percutaneous techniques. ${ }^{14-18}$ Percutaneous coronary intervention (PCI) should be considered in patients with localized proximal dissections in large vessels associated with ongoing ischemia or with a TIMI grade $0-1$ coronary flow. In patients with an evolving myocardial infarction (MI), an attempt to restore coronary flow is always justified. ${ }^{15}$ Stone and St Goar reported the use of primary angioplasty in this setting. ${ }^{28}$ However, $\mathrm{PCI}$ in patients with SCAD is associated with significant technical difficulties, including negotiating the wire through the true lumen, the risk of dissection or hematoma propagation, and side-branch occlusion. ${ }^{19-22}$ If PCI is contemplated, strategies to minimize complications include: (1) the use of intracoronary imaging to ensure true lumen wire position and guide procedures, (2) using direct stenting if feasible (for shorter lesions that can be covered by a single stent, ensuring adequate $5 \mathrm{~mm}$ edge coverage to accommodate hematoma extension), (3) stenting proximally to seal the "entry door", and (4) stenting of proximal and distal edges first before stenting the middle (Figure 1). ${ }^{13,15,17}$ Even if stent implantation is considered, a conservative approach should be favored, avoiding a "full-metal-jacket" strategy. ${ }^{15,19}$ Residual distal coronary dissection may sometimes be left untreated, provided there are no significant residual stenoses and the coronary flow is normal. ${ }^{19,25}$ Notably, spontaneous resolution of these distal residual dissections is frequently demonstrated at late follow-up. ${ }^{19}$ Whether drug-eluting stents (DES) provide any specific advantage over bare metal stents in the management of SCAD remains unclear. DES may be preferred for long dissected segments in order to reduce the risk of late restenosis. However, DES might also interfere with the normal healing process of the vessel wall, and late stent malapposition with resorption of intramural hematoma is a concern for very late stent thrombosis. More recently, the use of a cutting balloon has been advocated in selected cases to fenestrate the membrane and decompress the false lumen. ${ }^{29}$ Likewise the use of bioresorbable vascular scaffolds appears very attractive and promising in this setting because they eventually resorb completely from the vessel wall, thus facilitating full vessel restoration.

Alternatively, surgery should be considered in unstable patients with left main or 3-vessel involvement and also in patients with ischemia after a failed PCI. ${ }^{4-13,19-22}$ The major pitfalls of CABG in patients with SCAD are vessel frailty and the challenge of suture apposition in dissected arteries while performing anastomosis of grafts. Similarly, the results of large series of patients undergoing surgical revascularization with SCAD are lacking. Cardiac transplantation may be required in patients evolving with severe left ventricular dysfunction.

Earlier reports with aggregated data from retrospective analyses suggested that early revascularization was associated with improved clinical outcomes as compared with medical therapy alone. ${ }^{30}$ However, a "watchful waiting" strategy has been proposed by many investigators for stable patients with SCAD. Recent series demonstrated a good clinical outcome following a conservative medical management strategy in selected patients with SCAD. ${ }^{19-22}$ Indeed, most patients have a favorable prognosis following initial clinical stabilization. This strategy will be discussed in detail later. A flow diagram with a proposal for diagnostic and clinical management is presented in Figure 4.

\section{Diagnostic Value of Intracoronary Imaging Modalities}

As SCAD may be associated with significant acute morbidity, an early and accurate diagnosis remains of paramount importance. Until now, clinical diagnosis of SCAD has relied on the indirect visualization of a radiolucent intimal "flap" on angiography. ${ }^{14-18}$ In most cases, the angiographic narrowing caused by an intramural hematoma impinging into the lumen is misinterpreted as atherosclerotic disease (types 2 and 3 angiographic SCAD). ${ }^{14,15}$ Intravascular ultrasound (IVUS) and, more recently, optical coherence tomography (OCT), have become established as valuable novel intracoronary tools in the diagnosis of this entity. ${ }^{15}$ These techniques provide tomographic, high-resolution images of the coronary wall, and therefore, unique insights of the underlying substrate of patients with SCAD. ${ }^{15}$ The analogy with the diagnostic challenges in patients with aortic dissection is pertinent; ultimately, SCAD remains a disease of the vessel wall that can only be comprehensively analyzed using tomographic techniques. ${ }^{15}$

\section{IVUS}

Initially, IVUS findings were described from single-patient case-reports or very small case-series. ${ }^{31,32}$ IVUS gives a clear picture of the vessel wall (axial resolution $150 \mu \mathrm{m}$ ) and can differentiate the normal vessel wall or atherosclerotic plaque from SCAD. IVUS readily delineates the true and the false lumens and detects the presence of false lumen thrombosis. ${ }^{31,32}$ The shape and severity of true lumen compression can be also nicely visualized. Moreover, the full extent of the false lumen is readily depicted because ultrasound deeply penetrates the vessel wall, providing a complete picture of the entire vessel. ${ }^{31,32}$ Maehara et al ${ }^{31}$ described the IVUS findings in 5 patients who showed intramural hematoma occupying the dissected false lumen. All had just moderate "stenosis" on angiography without the typical radiolucent flap, and none showed intimal tears connecting the true and false lumens on IVUS. Arnold et al described 4 patients with SCAD in whom IVUS was used to confirm the presence of an intramural hematoma; similarly, an intimal tear could not be visualized. ${ }^{32}$

Subsequent studies confirmed and expanded on the value of IVUS in these patients. The normal 3-layered appearance of the vessel wall can be detected along the unaffected segments. Separation of the true lumen (relatively small and originating the take-off of the side-branches) from the false lumen (larger and more echogenic) is clearly visualized. The longitudinal and circumferential extension of the disease may be nicely depicted, even in segments without lumen compression. In most cases, the outer border of the vessel wall (external elastic lamina) can be visualized even in areas with large hematomas. The presence of thrombus in the false lumen can be also depicted. Patients with an intimal tear usually show echogenic stagnant contrast in the false lumen. However, because of inadequate nearfield resolution and the ring-down artifact, sometimes the dissection flap embracing the IVUS catheter may be 

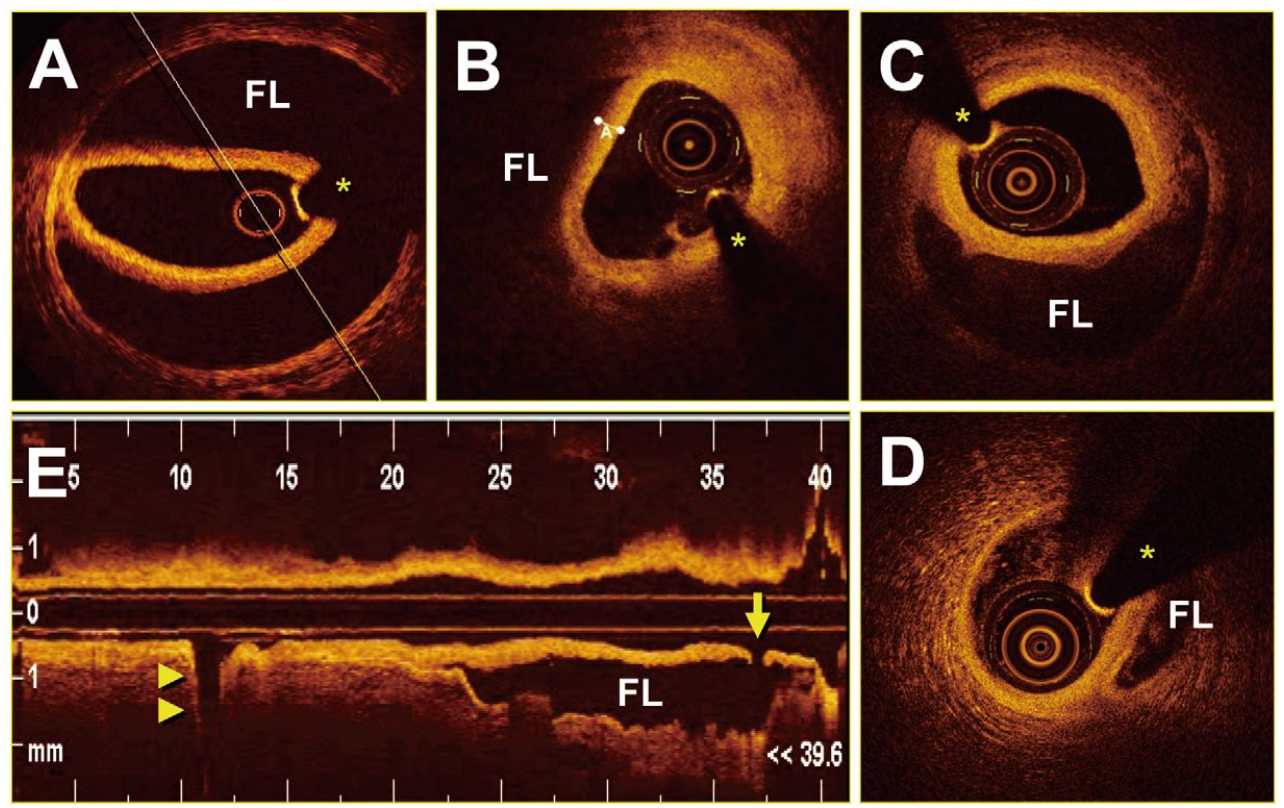

Figure 5. Optical coherence tomography images in patients with spontaneous coronary artery dissection. (A-D) Intima-medial membrane separating the true lumen from the false lumen (FL). (E) Longitudinal display disclosing the intimal rupture (arrow). $\left(^{*}\right)$ Wire artifact. Sidebranch exit (arrowheads). Reproduced from Alfonso $\mathrm{F}$ et al ${ }^{36}$ with permission from the American College of Cardiology Foundation.

difficult to visualize. Furthermore, in most patients the spatial resolution of IVUS remains suboptimal for reliably detecting the intimal rupture.

\section{OCT}

More recently, the value of OCT in the diagnosis of SCAD has been suggested by several single-patient case-reports or small series. ${ }^{33-36}$ These previous reports emphasized the value of the superb resolution of this technique $(15 \mu \mathrm{m})$ in the diagnosis of SCAD. In particular, OCT appears ideally suited to identify the "entry door" (intimal tear). ${ }^{33-36}$ This may have important therapeutic implications should an intervention be required, as this segment must be adequately sealed by the stent. ${ }^{15}$ Furthermore, the thickness of the dissecting membrane can be accurately measured. In fact, OCT has demonstrated that, in most patients, this membrane is relatively "thick" and actually corresponds to the intima and most of the medial layer (Figure 5). ${ }^{33-36}$

We performed a prospective study in which OCT was systematically used in patients $(n=17)$ with a clinical or angiographic suspicion of SCAD. ${ }^{36}$ In our series, OCT was of major value to exclude the presence of the disease in patients with normal coronary arteries but with a strong clinical suspicion of the disease, and also in patients with ambiguous angiographic findings. Indeed, in 6 patients presenting with a confined lumen narrowing or an angiographic linear filling defect, OCT was able to definitively rule out the presence of SCAD. All these patients presented with MI, and OCT disclosed intracoronary red thrombus or severe atherosclerotic plaque. Interestingly, a superficial, non-occlusive, calcified nodule was found in a patient, who eventually evolved into a case of Takotsubo cardiomyopathy. Our findings demonstrate that OCT is of major value to rule out the diagnosis of SCAD in patients with images mimicking this entity. This is clinically relevant because an early definitive diagnosis allows adequate triage to either PCI or aggressive antithrombotic therapy. ${ }^{36}$

More importantly, in 11 patients, OCT was instrumental in confirming the presence of SCAD and provided unique insights into the underlying substrate. ${ }^{36}$ All these patients presented with acute MI and severe lumen compromise, but only 3 had the classical angiographic intimal flap. OCT readily visualized the affected coronary wall along the entire vessel. The total length of the diseased segment was $32 \pm 12 \mathrm{~mm}$ (double lumen length $8.4 \pm 6.7 \mathrm{~mm}$; intramural hematoma length $21.9 \pm 15 \mathrm{~mm}$ ). In most of the patients, the dissecting membrane was relatively thick (mean $350 \mu \mathrm{m}$ ), suggesting that it actually consisted of "intima plus media". These findings are consistent with those of classical pathological studies. In addition, the 2 lumens (true and false) were readily identified. The circumferential and longitudinal extent of the disease and the involvement of related side-branches could be easily studied. Associated thrombus was detected in most cases, although in many patients the shadowing caused by red thrombus partially obscured the vessel wall. In addition, thrombosis of the false lumen was detected in many cases. Finally, the "entry door" (confined intimal tear) could be recognized in 7 of the 11 patients. Interestingly, the thinnest part of the intima-medial membrane tended to be located close to the intimal tear. ${ }^{36}$

In our experience, OCT is also of major help in patients with SCAD requiring stent implantation (Figure 6). ${ }^{36}$ Before stenting, OCT is critical for confirming that the guidewire is indeed located within the true lumen. Obviously, implanting a stent in the false lumen can have catastrophic consequences. Furthermore, OCT may guide these interventions. First, OCT may ensure that the intimal tear (entry door) has been adequately sealed by the stent. Second, the length of the stent may 

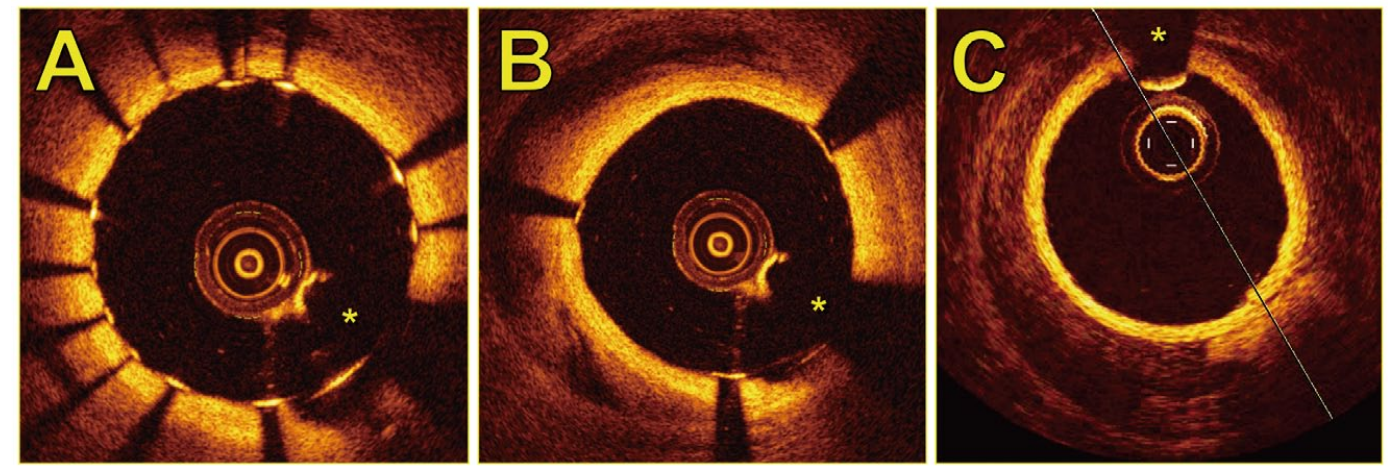

Figure 6. Optical coherence tomography images after stenting. (A) Excellent expansion and apposition of the stent struts. (B) Residual abluminal hematoma after stenting. (C) Residual intramural hematoma distal to the stented segment. Reproduced from Alfonso $\mathrm{F}$ et $\mathrm{al}^{36}$ with permission from the American College of Cardiology Foundation.

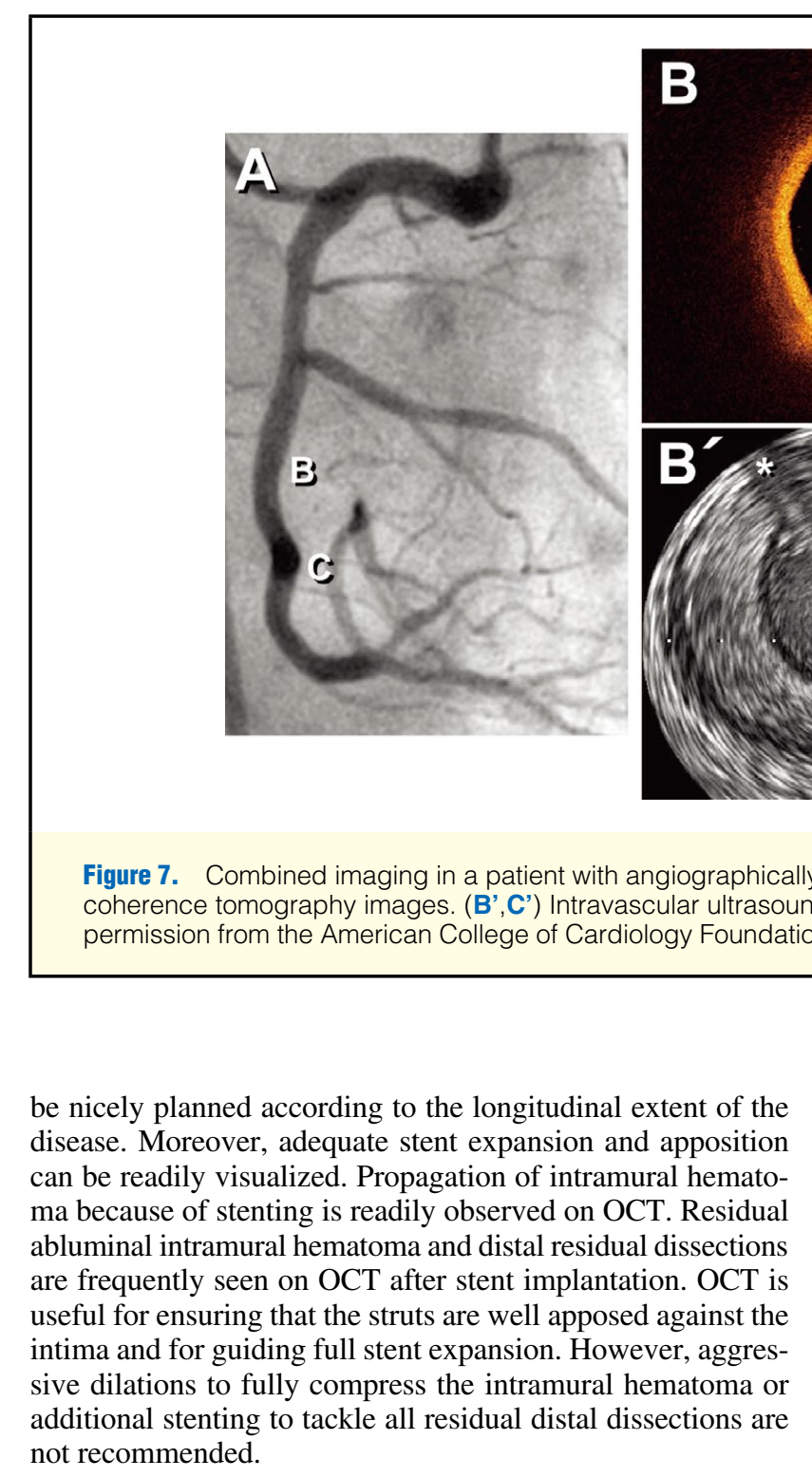

\section{Combined Use of IVUS and OCT}

Poon et al suggested the potential complementary value of a combined interrogation using IVUS and OCT in 1 patient with SCAD. ${ }^{37}$ We performed a prospective study systematically assessing the value of this combined imaging modality strategy in patients with SCAD (Figure 7). ${ }^{38,39}$ We found that OCT had a higher diagnostic value for detecting the precise location of the intimal tear and also for accurately measuring the thickness of the intima-medial membrane. In our study, OCT provided crisp images of the intimal-lumen interface and allowed more precise measurements of the true lumen. OCT disclosed even minor irregularities in the coronary lumen and readily 
detected tiny intracoronary thrombi that were not seen with IVUS. ${ }^{39}$ Nevertheless, in most cases the outer circumference of the vessel wall was not recognized on OCT because of shadowing/attenuation issues, or as a result of limited field of view. Furthermore, in some cases a complete blood-free lumen was not obtained because of intentional use of careful and gentle flushing sequences in these vulnerable vessels. ${ }^{38,39} \mathrm{In}$ addition, in cases of severe SCAD the OCT catheter may completely occlude the true lumen, affecting the clearance of residual blood and impeding vessel wall visualization. Conversely, an obstructive IVUS catheter will still provide adequate images of the vessel wall. IVUS enabled better delineation of the external elastic lamina and nicely visualized the entire diseased segment. In addition, the presence of thrombus in the false lumen and its heterogeneous scatter was better visualized with IVUS than with OCT. On IVUS, most sidebranches emerging from the true lumen showed a characteristic echogenic pattern and could be traced for some distance outside the main vessel. It is important to keep in mind that red thrombus does not obscure the underlying vessel wall on IVUS. Images consistent with intimal ruptures were only identified with IVUS in 2 patients and these images were not as sharp or clear as those depicted with OCT. ${ }^{39}$

Although intracoronary techniques provide strikingly beautiful images, they should be used with care to avoid further vessel injury. ${ }^{31-39}$ In cases of diffusely diseased segments in small or distal vessels, imaging should be restricted to the proximal segment of the dissected artery. Likewise, deep engagement of the guiding catheter should be avoided and gentle injection intensity selected to prevent any deleterious increase in coronary pressure. Finally, it is important to keep in mind that the use of combined intracoronary imaging remains investigational $\mathrm{l}^{31-39}$ and is costly; most laboratories can only utilize 1 intracoronary imaging modality to confirm SCAD diagnosis.

Intracoronary tomographic techniques have been able to unravel the "dark side of the moon", of this entity emphasizing the challenges of angiographic diagnosis of SCAD. ${ }^{15}$ In our experience, intramural hematomas should be suspected in arteries with angiographic diffuse luminal narrowing, especially in the setting of arteries with "curly" or "corkscrew" pattern on the rest of the vessel, a "straightening" appearance or a "broken line" vessel morphology. ${ }^{15,16}$ As SCAD usually heals over time, confirming a complete "restitutio ad integrum" may require the unique insights obtained by intracoronary imaging.

\section{Association With FMD}

FMD is another elusive condition that recently was linked to SCAD. ${ }^{15}$ FMD is a non-inflammatory, non-atherosclerotic vascular disease of unknown etiology. ${ }^{40-43}$ It tends to affect young females and usually presents as arterial stenosis, aneurysms, dissection or thrombosis. ${ }^{40-43}$ Although multiple noncoronary beds can be involved, the renal and carotid arteries are most often affected. From a histopathological perspective, FMD has been categorized into intimal fibroplasia, medial fibroplasia, medial hyperplasia, perimedial fibroplasia, and adventitial fibroplasia. ${ }^{40-43}$ Medial fibroplasia is the most common abnormality, accounting for up to $90 \%$ of cases. The characteristic angiographic pattern consists of alternating areas of thinned and thickened medial collagen ridges, with stenotic arterial segments followed by post-stenotic dilation, resulting in the "string of beads" appearance that is the hallmark of this disease. ${ }^{40-43}$ The angiographic appearance depends on the histopathologic subtype, with the most common subtype of medial fibroplasia appearing as the "string of beads" morphology where the beads are larger than the normal caliber of the artery. Rare FMD subtypes include intimal fibroplasia (presenting angiographically as focal or tubular lesions) and perimedial fibroplasia (with beads smaller than the reference vessel). ${ }^{40-43}$

Despite all these angiographic findings, FMD remains an under-diagnosed condition, mainly because most manifestations are clinically silent and only discovered incidentally when imaging is performed for another indication. ${ }^{42}$ There has been little progress in the understanding of the epidemiology, pathogenesis, and outcomes since its first description back in 1938. ${ }^{40-43}$ Recently, Olin et $\mathrm{al}^{42}$ reviewed clinical features, presenting symptoms, and vascular events in the first 447 patients enrolled in a national-level FMD-registry including 9 US sites, which represents the largest cohort of patients with FMD to date. The disease frequently affected medium-sized arteries. A past or present history of vascular events was common: $19 \%$ of patients had a transient ischemic attack or stroke, $20 \%$ had experienced arterial dissection, and $17 \%$ had aneurysms. Most patients $(91 \%)$ were women and mean age at diagnosis was 52 years. The most frequent indications for therapy were hypertension, headache, tinnitus and dizziness but also aneurysms, and dissections. ${ }^{42}$ In this large registry, only 8 patients (1.9\%) had an acute MI. A total of 88 patients $(20 \%)$ presented with dissections but of these, only in $3(3.4 \%)$ did dissections involve the coronary arteries.

Surprisingly, coronary artery FMD has rarely been reported pathologically in small vessels, including the coronary arteries, although the condition has been reported as a rare cause of MI and sudden death. ${ }^{44}$ FMD was previously infrequently associated with SCAD and until very recently, the clinical relevance of this association was buried within the abundant list of diseases associated with SCAD. ${ }^{14-18}$ In an autopsy series of 50 patients with sudden cardiac death, Hill and Sheppard found 2 patients who died of coronary FMD and 8 patients who died of SCAD. ${ }^{45}$

The Canadian group from Vancouver ${ }^{46-48}$ was the first to describe a clear association between FMD and SCAD. In an initial report, they described 7 perimenopausal women with acute MI who had isolated diffuse obliterative lesions in the mid and distal segments of the coronary arteries with coincidental renal FMD. ${ }^{46}$ The women had well-defined, abrupt transition to long lesions in the middle or distal coronary segments, and the remaining coronary segments were angiographically normal. The authors proposed that coronary FMD was a potential etiological factor for these ischemic events and also suggested that superimposed thrombus or dissection might act as contributing factors. In the absence of intracoronary imaging, they initially proposed that the diffuse stenotic angiographic appearance was related to coronary artery FMD. In a later series of 6 women presenting with MI and somewhat unusual angiographic appearance (including types 2 and 3 SCAD angiographic appearances), Saw et al were able to make the definitive diagnosis using OCT or IVUS that these epicardial stenoses were in fact intramural hematoma (unrecognized SCAD $)^{47}$ in these patients who all had coincidental FMD in other vascular territories. The characteristics of intracoronary FMD were also described using OCT or IVUS. ${ }^{48}$ Subsequently, Saw et $\mathrm{al}^{22}$ expanded their initial observations to a larger series of 50 patients with non-atherosclerotic SCAD. Patients were systematically screened for noncoronary FMD with invasive or noninvasive angiography in 3 vascular territories (renal, iliac and cerebrovascular). They found FMD in at least 1 noncoronary territory in most patients with SCAD. Renal artery screening was performed in $94 \%$ of patients, iliac artery screening was performed in $64 \%$ of patients, and cerebrovas- 


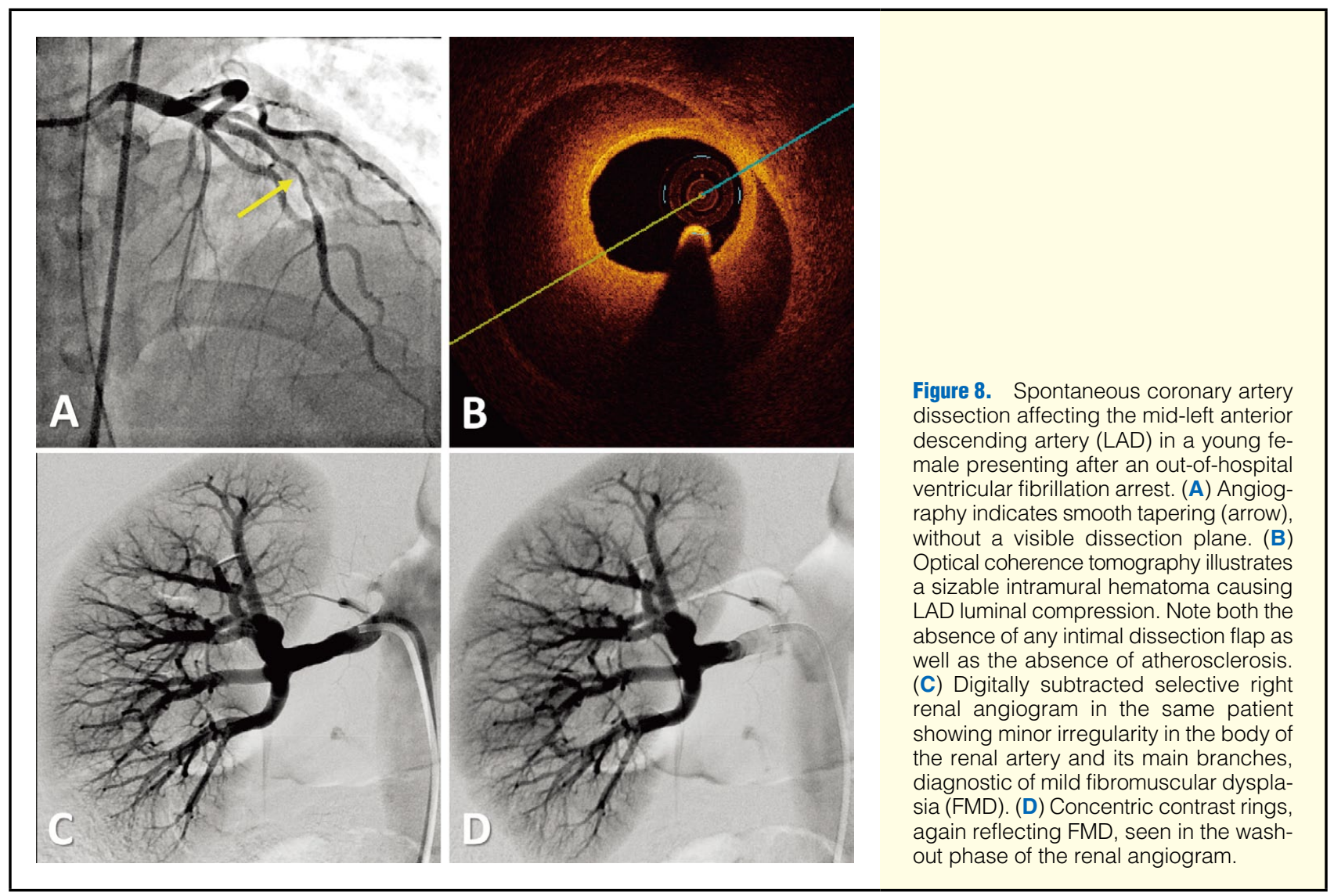

cular screening was performed in $66 \%$ of patients. Notably, FMD was identified in at least 1 noncoronary vascular territory in $86 \%$ of screened patients and nearly half of them had FMD in more than 1 noncoronary vascular territory. That study demonstrated the superior diagnostic accuracy of invasive angiography over noninvasive imaging techniques for the identification of FMD. Of interest, among the 43 patients with FMD, $58 \%$ had renal artery involvement, $49 \%$ iliac artery involvement, and $47 \%$ had cerebrovascular involvement ( 7 with cerebral or carotid aneurysms). Accordingly, the authors proposed that the prevalence of noncoronary FMD among SCAD patients was too high to be merely coincidental. ${ }^{46-48}$ This uniquely high prevalence of the association implies a likely causative link (Figure 3). ${ }^{46-48}$

Notably, this association has also been confirmed by other groups. Toggweiler et al from Switzerland ${ }^{49}$ screened 12 SCAD patients with whole-body magnetic resonance angiography (MRA) and duplex sonography and found 3 patients (25\%) with renal FMD ( 2 with findings of FMD and 1 with spontaneous dissection of a renal artery). The lower prevalence in their study may be related to the limited diagnostic sensibility of MRA and MSCT to identify mild forms of FMD. More recently, however, a study from the Mayo Clinic $^{21}$ reported that up to $50 \%$ of the patients with SCAD undergoing iliofemoral angiography also had incidental external iliac FMD. Although these findings were identified in patients with available femoral angiograms for other indications, these authors also suggested that FMD could indeed represent a potentially causative factor for SCAD. Finally, another recent report also from the Mayo Clinic $^{50}$ described the value of noninvasive CTangiography in patients with SCAD. Extracoronary vascular abnormalities were detected in 27 of 39 patients (69\%) and included FMD, aneurysms, dissection, and aortic tortuosity, seen in the iliac (36\%), carotid and/or vertebral (31\%), splanchnic $(10 \%)$, and renal $(26 \%)$ arteries and in the thoracic and/or abdominal aorta (10\%) (Figure 8).

The presence of FMD should direct clinicians towards a diagnosis of SCAD in patients with ambiguous coronary angiograms. ${ }^{15,51}$ Furthermore, in selected patients the use of tomographic techniques may be of major value to further unravel the underlying lesional substrate in the coronary arteries and other larger noncoronary arterial beds. ${ }^{52}$ Altogether, current evidence suggests that the striking prevalence of FMD in patients with SCAD might implicate causation. However, this persuasive hypothesis should be further investigated to fully establish the predisposing pathologic substrate of this elusive and challenging clinical entity. ${ }^{46-48,51}$

\section{Recent Large Series of Patients With SCAD}

As previously discussed, earlier studies of this rare disease were from case-report series or small, retrospective observational studies. More recently, however, our current knowledge has been enriched by data from larger SCAD series.

We carefully designed a prospective clinical protocol to assess the clinical, angiographic, analytical and prognostic findings in a large series of patients with SCAD. ${ }^{19}$ The protocol was based on the central idea of offering an initial conservative medical management strategy for patients presenting with SCAD. Therefore, revascularization was only considered in cases of ongoing or recurrent ischemia. In addition, a high level of clinical suspicion was maintained during the study 
period and additional diagnostic tests were used as required in cases of equivocal or elusive angiographic diagnosis. In this regard, intracoronary imaging, including IVUS or OCT, was systematically considered in patients with a strong clinical suspicion of SCAD but who presented with either angiographically normal coronary arteries or confined lumen narrowing but otherwise smooth coronary vessels. Moreover, these invasive imaging modalities were also used in many patients with a clear angiographic diagnosis of SCAD in order to give additional diagnostic insights regarding the pathophysiology, severity and extent of the disease, to decide upon the need of coronary revascularization and, eventually, to guide the interventions. Likewise, an exhaustive analysis of multiple inflammatory and immunological markers was systematically obtained. Finally, a detailed extended clinical follow-up was organized for all these patients. Furthermore, in selected cases, clinical follow-up was completed by angiographic follow-up or, alternatively, with noninvasive diagnostic imaging techniques. ${ }^{19}$

The cohort consisted of 45 consecutive patients with SCAD who were studied during a 6-year period. ${ }^{19}$ The estimated incidence of SCAD was $0.27 \%$, as assessed from consecutive patients undergoing a first diagnostic $\mathrm{CAG}$ at our institution in Madrid during the study period. Of these 45 patients, 27 $(60 \%)$ had "isolated" SCAD and 18 had SCAD associated with CAD. Mean age was $53 \pm 11$ years and 26 patients $(58 \%)$ were female. Most patients (76\%) presented with an acute MI. The LAD was frequently involved (half of the patients) and the disease showed a diffuse angiographic pattern (mean lesion length $31 \pm 23 \mathrm{~mm}$ ). Using the prespecified conservative management strategy, only one-third of patients (16 [35\%]) required coronary revascularization during admission; 9 patients underwent revascularization immediately after the angiographic diagnosis of SCAD because of ongoing ischemia, and 7 additional patients underwent delayed revascularization because of recurrent ischemia during hospitalization. Revascularization procedures included coronary surgery in 2 patients and stent implantation in 14 . In 7 of the 14 patients treated with coronary stenting, residual non-flow limiting dissections were intentionally left untreated distal to the stented segment. One patient died in relation to surgery but no additional patient died or suffered a recurrent MI. Notably, despite our exhaustive analytic protocol, no significant inflammatory or immunological abnormalities were detected in this series. Of interest, at late clinical follow-up (median 2 years) only 3 patients had developed adverse events (1 patient died from congestive heart failure and 2 required revascularization). However, no patient suffered an MI or sudden death during follow-up. The event-free survival at 3 years was similar (94\% and $88 \%$, respectively) for patients with isolated SCAD and SCAD associated with CAD. Interestingly, at late angiographic followup (median 9 months) SCAD had completely resolved spontaneously in $50 \%$ of the patients and improved in most of the remaining patients. The findings of this prospective and relatively large series of patients with late clinical follow-up suggest that in patients with SCAD, the use of a "conservative" therapeutic strategy (watchful waiting strategy) provides an excellent acute and long-term clinical outcome. This favorable clinical outcome, together with the demonstration of spontaneous disappearance of the dissection image on angiography reinforces the idea that, whenever possible, a conservative initial approach is indicated in these patients. ${ }^{15,19}$

As compared with patients with conventional CAD, the SCAD patients in our series were relatively young and frequently female. ${ }^{19}$ However, male and adult patients were also found. Young, female patients were significantly more frequent in the group of isolated SCAD as compared with that of SCAD associated with CAD. In addition, a relative high rate of classical coronary risk factors was found, especially in patients with associated CAD. Surprisingly, however, only 1 patient in this series presented in the postpartum period, another had early menopause and a third patient was taking oral contraceptives. This suggests that although these classical risk factors may elicit SCAD, they remain rare among unselected patients with the diagnosis of SCAD. Most patients presented with an acute MI but STEMI occurred more often in patients with isolated SCAD. ${ }^{19}$

To summarize, the main findings of the Madrid study are as follows: (1) SCAD is a serious clinical condition with a high initial morbidity, mainly related to the presentation as acute MI; (2) once the diagnosis of SCAD is established, a "conservative" management strategy, restricting revascularization for cases of ongoing/recurrent ischemia, is associated with an excellent long-term prognosis; (3) in patients with a clinical suspicion of SCAD, angiography often fails to establish the diagnosis but intracoronary imaging remains instrumental in disclosing the presence of the disease; (4) last but not least, SCAD may spontaneously heal over time, reinforcing the idea of selecting a conservative initial strategy whenever possible.

Saw et al reported on a large Canadian series of 50 patients with SCAD prospectively and retrospectively identified over 6 years at Vancouver General Hospital. ${ }^{22}$ Mean age was 51 years, and $98 \%$ of patients were women. All presented with MI, 30\% had STEMI and 70\% non-STEMI. Only 1 patient was postpartum, and 2 were involved in intense isometric exercising. Interestingly, however, emotional stress was reported in $26 \%$ of cases. Many patients were postmenopausal (45\%), had a history of migraines (27\%) or depression (29\%). Revascularization was only needed in 9 patients (18\%) (7 coronary stenting, 2 coronary surgery) and no patient died during hospitalization. Indeed, early discharge $(2.7 \pm 2.6$ days $)$ was possible in most patients. This excellent clinical outcome also suggests that a watchful waiting strategy is reasonable for "stabilized" patients with SCAD.

Finally, Tweet et al, from the Mayo Clinic, reviewed the characteristics, clinical management and outcome of the largest cohort of patients with SCAD ever reported to date. ${ }^{20,21}$ They analyzed 87 consecutive patients with SCAD recruited during 3 decades and estimated an annual incidence of $0.26 / 100,000$ persons, but also cautioned that this figure was likely an underestimation. Presentation as sudden death, under-referral in otherwise healthy young women, and underrecognition by angiography remain important factors leading to underdiagnosis. All these patients (mean age 43 years, $82 \%$ female) had a "dissection plane" on CAG, which suggests that patients with isolated intramural hematoma were not included in their series. Interestingly, predisposing conditions involved extreme exertion ( $44 \%$ of males) and postpartum status $(18 \%$ of females). Clinical presentation included STEMI in half of the patients, but $14 \%$ presented with malignant ventricular arrhythmias. In this large series, up to two-thirds of patients underwent coronary revascularization during initial hospitalization, but the results of these interventions were relatively poor. Of concern, unexpected disease propagation occurred in $16 \%$ of patients. Despite the adoption of a rather optimistic definition for success (patent artery when the vessel was totally occluded, a TIMI 2/3 flow in the remaining patients) that allowed multiple/unplanned stent implantation, overall technical success of PCI was only $65 \%$ (28/43 procedures). Likewise, in this series the results of surgical revascularization 
were also rather disappointing. ${ }^{21}$ Indeed, although early results of CABG were apparently favorable, most arterial conduits were eventually occluded at late angiographic surveillance. Interestingly, in this series from the Mayo Clinic, SCAD recurred in 15 patients during late clinical follow-up. ${ }^{21}$ Recurrence rates have been much lower in other previous series. Of note, when the results of patients with SCAD were compared with those of a matched series of patients with ACS from atherosclerotic CAD, a better outcome was found in the SCAD group. $^{21}$

Altogether, these results again suggest that revascularization should be reserved for selected patients with SCAD, including those with persistent symptoms or who are considered to be at high-risk providing coronary anatomy is considered favorable (Figure 4).

\section{Conclusions}

In summary, SCAD is a relatively infrequent condition that has been under-diagnosed. SCAD predominantly affects women in their 5th and 6th decades, although younger women also may be affected. Diagnosis remains challenging because CAG is imperfect, but an angiographic classification and algorithm has been described recently to improve diagnosis and management. Adjunct intracoronary imaging with OCT or IVUS can be instrumental in confirming the diagnosis of SCAD in cases of angiographic ambiguity. SCAD has also been linked to many noncoronary arteriopathies, the most important association being FMD, which is present in the majority of patients with SCAD. In general, a conservative approach avoiding revascularization for stable SCAD patients is advocated by most experts. However, PCI or CABG may be required in patients with ongoing ischemia or critical anatomy involvement (eg, left main). Medical management of SCAD patients remain controversial without randomized trial data to guide therapy. Future studies are warranted to address the link between SCAD and FMD and to elucidate the long-term prognosis and the indications for revascularization in this challenging clinical entity.

\section{Disclosures}

Authors have nothing to disclose in relation to this manuscript.

\section{References}

1. Pretty H. Dissecting aneurysms of coronary artery in woman aged 42: Rupture. BMJ 1931; 1: 667.

2. Davies MJ, Treasure T, Richardson PD. The pathogenesis of spontaneous arterial dissection. Heart 1996; 75: 434-435.

3. Forker AD, Rosenlof RC, Weaver WF, Carveth SW, Reese HE. Primary dissecting aneurysm of the right coronary artery with survival. Chest 1973; 64: 656-658.

4. DeMaio SJ Jr, Kinsella SH, Silverman ME. Clinical course and longterm prognosis of spontaneous coronary artery dissection. Am J Cardiol 1989; 64: 471-474.

5. Jorgensen MB, Aharonian V, Mansukhani P, Mahrer PR. Spontaneous coronary dissection: A cluster of cases with this rare finding. Am Heart J 1994; 127: $1382-1387$.

6. Hering D, Piper C, Hohmann C, Schultheiss HP, Horstkotte D. Prospective study of the incidence, pathogenesis and therapy of spontaneous, by coronary angiography diagnosed coronary artery dissection. Z Kardiol 1998; 87: 961 -970 (in German).

7. Celik SK, Sagcan A, Altintig A, Yuksel M, Akin M, Kultursay H. Primary spontaneous coronary artery dissections in atherosclerotic patients: Report of nine cases with review of the pertinent literature. Eur J Cardiothorac Surg 2001; 20: 573-576.

8. Thompson EA, Ferraris S, Gress T, Ferraris V. Gender differences and predictors of mortality in spontaneous coronary artery dissection: A review of reported cases. J Invasive Cardiol 2005; 17: 5961.

9. Butler R, Webster MW, Davies G, Kerr A, Bass N, Armstrong G, et al. Spontaneous dissection of native coronary arteries. Heart 2005;
91: $223-224$.

10. Vanzetto G, Berger-Coz E, Barone-Rochette G, Chavanon O, Bouvaist $\mathrm{H}$, Hacini R, et al. Prevalence, therapeutic management and mediumterm prognosis of spontaneous coronary artery dissection: Results from a database of 11,605 patients. Eur J Cardiothorac Surg 2009; 35: $250-254$.

11. Mortensen KH, Thuesen L, Kristensen IB, Christiansen EH. Spontaneous coronary artery dissection: A Western Denmark heart registry study. Catheter Cardiovasc Interv 2009; 74: 710-717.

12. Motreff P, Souteyrand G, Dauphin C, Eschalier R, Cassagnes J, Lusson JR. Management of spontaneous coronary artery dissection: Review of the literature and discussion based on a series of 12 young women with acute coronary syndrome. Cardiology 2010; 115: 10-18.

13. Walsh S, Jokhi P, Saw J. Successful percutaneous management of coronary dissection and extensive intramural haematoma associated with ST elevation MI. Acute Cardiac Care 2008; 10: 231-233.

14. Vrints CJ. Spontaneous coronary artery dissection. Heart 2010; 96: $801-808$.

15. Alfonso F. Spontaneous coronary artery dissection: New insights from the tip of the iceberg? Circulation 2012; 126: 667-670.

16. Saw J. Coronary angiogram classification of spontaneous coronary artery dissection. Catheter Cardiovasc Interv 2013 November 13, doi:10.1002/ccd.25293.

17. Saw J. Spontaneous coronary artery dissection. Can J Cardiol 2013; 29: $1027-1033$.

18. Giacoppo D, Capodanno D, Dangas G, Tamburino C. Spontaneous coronary artery dissection. Int J Cardiol 2014; 175: 8-20.

19. Alfonso F, Paulo M, Lennie V, Dutary J, Bernardo E, JiménezQuevedo P, et al. Spontaneous coronary artery dissection: Long-term follow-up of a large series of patients prospectively managed with a "conservative" therapeutic strategy. JACC Cardiovasc Interv 2012; 5: $1062-1070$.

20. Tweet MS, Gulati R, Aase LA, Hayes SN. Spontaneous coronary artery dissection: A disease-specific, social networking communityinitiated study. Mayo Clin Proc 2011; 86: 845-850.

21. Tweet MS, Hayes SN, Pitta SR, Simari RD, Lerman A, Lennon RJ, et al. Clinical features, management, and prognosis of spontaneous coronary artery dissection. Circulation 2012; 126: 579-588.

22. Saw J, Ricci D, Starovoytov A, Fox R, Buller CE. Spontaneous coronary artery dissection: Prevalence of predisposing conditions including fibromuscular dysplasia in a tertiary center cohort. JACC Cardiovasc Interv 2013; 6: 44-52.

23. Borczuk AC, van Hoeven KH, Factor SM. Review and hypothesis: The eosinophil and peripartum heart disease (myocarditis and coronary dissection): Coincidence or pathogenetic significance? Cardiovasc Res 1997; 33: 527-532.

24. Moreno R, Pérez del Todo J, Nieto M, Alba F, Alfonso F, GarciaRubira JC, et al. Primary stenting in acute myocardial infarction secondary to right coronary artery dissection following blunt chest trauma: Usefulness of intracoronary ultrasound. Int J Cardiol 2005; 103: $209-211$.

25. Alfonso F, Hernandez R, Goicolea J, Segovia J, Perez-Vizcayno MJ, Bañuelos $\mathrm{C}$, et al. Coronary stenting for acute coronary dissection after coronary angioplasty: Implications of residual dissection. J Am Coll Cardiol 1994; 24: 989-995.

26. Ohlmann P, Weigold G, Sang-Wook K, Hassani SE, Escolar E, Pichard $\mathrm{AD}$, et al. Spontaneous coronary dissection: Computed tomography appearance and insights from intravascular ultrasound examination. Circulation 2006; 113: e403-e405, doi:10.1161/CIRCULATIONAHA. 105.572313 .

27. Das Neves BC, Núñez-Gil IJ, Alfonso F, Hernández R, Cuevas C, Jimenez Quevedo P, et al. Evolutive recanalization of spontaneous coronary artery dissection: Insights from a multimodality imaging approach. Circulation 2014; 129: 719-720.

28. Stone GW, St Goar FG. Spontaneous coronary dissection resulting in acute myocardial infarction: Successful treatment with primary angioplasty. Cathet Cardiovasc Diagn 1996; 38: 62-66.

29. Yumoto K, Sasaki H, Aoki H, Kato K. Successful treatment of spontaneous coronary artery dissection with cutting balloon angioplasty as evaluated with optical coherence tomography. JACC Cardiovasc Interv 2014; 7: 817-819.

30. Shamloo BK, Chintala RS, Nasur A, Ghazvini M, Shariat P, Diggs JA, et al. Spontaneous coronary artery dissection: Aggressive vs. conservative therapy. J Invasive Cardiol 2010; 22: 222-228.

31. Maehara A, Mintz GS, Castagna MT, Pichard AD, Satler LF, Waksman $\mathrm{R}$, et al. Intravascular ultrasound assessment of spontaneous coronary artery dissection. Am J Cardiol 2002; 89: 466-468.

32. Arnold JR, West NEJ, van Gaal WJ, Karamitsos TD, Banning AP. The role of intravascular ultrasound in the management of spontaneous coronary artery dissection. Cardiovasc Ultrasound 2008; 6: 24. 
33. Alfonso F, Canales E, Aleong G. Spontaneous coronary artery dissection: Diagnosis by optical coherence tomography. Eur Heart J 2009; 30: 385.

34. Ishibashi K, Kitabata H, Akasaka T. Intracoronary optical coherence tomography assessment of spontaneous coronary artery dissection. Heart 2009; 95: 818.

35. Iglesias D, Salinas P, Jiménez-Valero S. Spontaneous coronary artery dissection evaluated by optical coherence tomography. $J$ Cardiovasc Med (Hagerstown) 2011; 12: 743-744.

36. Alfonso F, Paulo M, Gonzalo N, Dutary J, Jimenez-Quevedo P, Lennie $\mathrm{V}$, et al. Diagnosis of spontaneous coronary artery dissection by optical coherence tomography. J Am Coll Cardiol 2012; 59: $1073-1079$

37. Poon K, Bell B, Raffel OC, Walters DL, Jang IK. Spontaneous coronary artery dissection: Utility of intravascular ultrasound and optical coherence tomography during percutaneous coronary intervention. Circ Cardiovasc Interv 2011; 4: e5-e7, doi:10.1161/ CIRCINTERVENTIONS.110.959593.

38. Alfonso F, Paulo M, Dutary J. Endovascular imaging of angiographically invisible spontaneous coronary artery dissection. JACC Cardiovasc Interv 2012; 5: 452-453.

39. Paulo M, Sandoval J, Lennie V, Dutary J, Medina M, Gonzalo N, et al. Combined use of OCT and IVUS in spontaneous coronary artery dissection. JACC Cardiovasc Imaging 2013; 6: 830-832.

40. Slovut DP, Olin JW. Fibromuscular dysplasia. N Engl J Med 2004; 350: $1862-1871$

41. Saw J, Aymong E, Mancini GB, Sedlak T, Starovoytov A, Ricci D. Nonatherosclerotic coronary artery disease in young women. Can J Cardiol 2014; 30: 814-819.

42. Olin JW, Froehlich J, Gu X, Bacharach JM, Eagle K, Gray BH, et al. The United States Registry for Fibromuscular Dysplasia: Results in the first 447 patients. Circulation 2012; 125: 3182-3190.
43. Olin JW, Gornik HL, Bacharach JM, Biller J, Fine LJ, Gray BH, et al. Fibromuscular dysplasia: State of the science and critical unanswered questions: A scientific statement from the American Heart Association. Circulation 2014; 129: 1048-1078.

44. Lie JT, Berg KK. Isolated fibromuscular dysplasia of the coronary arteries with spontaneous dissection and myocardial infarction. Hum Pathol 1987; 18: 654-656.

45. Hill SF, Sheppard MN. Non-atherosclerotic coronary artery disease associated with sudden cardiac death. Heart 2010; 96: 1119-1125.

46. Pate GE, Lowe R, Buller E. Fibromuscular dysplasia of the coronary and renal arteries? Catheter Cardiovasc Interv 2005; 64: 138-145.

47. Saw J, Poulter R, Fung A, Wood D, Hamburger J, Buller CH. Spontaneous coronary artery dissection in patients with fibromuscular dysplasia: A case series. Circ Cardiovasc Interv 2012; 5: 134-137.

48. Saw J, Poulter R, Fung A. Intracoronary imaging of coronary fibromuscular dysplasia with OCT and IVUS. Catheter Cardiovasc Interv 2013; 82: E879-E883, doi:10.1002/ccd.24640.

49. Toggweiler S, Puck M, Thalhammer C, Manka R, Wyss M, Bilecen $\mathrm{D}$, et al. Associated vascular lesions in patients with spontaneous coronary artery dissection. Swiss Med Wkly 2012; 142: w13538.

50. Liang JJ, Prasad M, Tweet MS, Hayes SN, Gulati R, Breen JF, et al. A novel application of CT angiography to detect extracoronary vascular abnormalities in patients with spontaneous coronary artery dissection. J Cardiovasc Comput Tomogr 2014; 8: 189-197.

51. Alfonso F, Paulo M, Lennie V, Das-Neves B, Echavarría-Pinto M. Fibromuscular dysplasia and spontaneous coronary artery dissection: Coincidental association or causality? JACC Cardiovasc Interv 2013; 6: 638

52. Bastante T, Alfonso F. Insights of optical coherence tomography in renal artery fibrodysplasia in a patient with spontaneous coronary artery dissection. Arq Bras Cardiol 2014; 103: e18-e19, doi: 10.5935/abc. 20140100 . 